\title{
The Equivalence of Half-Quadratic Minimization and the Gradient Linearization Iteration
}

\author{
Mila Nikolova* $\quad$ Raymond Chan $^{\dagger}$
}

\begin{abstract}
A popular way to restore images comprising edges is to minimize a cost-function combining a quadratic data-fidelity term and an edge-preserving (possibly nonconvex) regularization term. Mainly because of the latter term, the calculation of the solution is slow and cumbersome. Half-quadratic (HQ) minimization (multiplicative form) was pioneered by Geman and Reynolds (1992) in order to alleviate the computational task in the context of image reconstruction with non-convex regularization. By promoting the idea of locally homogeneous image models with a continuous-valued line process, they reformulated the optimization problem in terms of an augmented cost function which is quadratic with respect to the image and separable with respect to the line process. Hence the name "half quadratic". Since then, a large amount of papers were dedicated to HQ minimization and important results including edge-preservation along with convex regularization and convergence have been obtained.

In this paper we show that HQ minimization (multiplicative form) is equivalent to the most simple and basic method where the gradient of the cost-function is linearized at each iteration step. In fact, both methods give exactly the same iterations. Furthermore, connections of HQ minimization with other methods, such as the quasi-Newton method and the generalized Weiszfeld's method, are straightforward.
\end{abstract}

Keywords: half-quadratic regularization, inverse problems, gradient linearization, optimization, signal and image restoration, variational methods.

\section{Introduction}

Let data $y \in \mathbb{R}^{q}$ be obtained from an original unknown image or signal $x^{*}$ via $y=A x^{*}+$ noise where $A \in \mathbb{R}^{q \times p}$ is a linear transform. This simple model addresses various applications such as denoising, deblurring, image reconstruction in tomography and other inverse problems $[1,2,3]$. Since $[4,5,6]$, the sought-after $\hat{x} \in \mathbb{R}^{p}$ is defined as the minimizer of an objective function $J: \mathbb{R}^{p} \rightarrow \mathbb{R}$ of the form

$$
\begin{aligned}
J(x) & =\|A x-y\|^{2}+\beta \Phi(x), \\
\Phi(x) & =\sum_{i=1}^{r} \varphi\left(\left\|G_{i} x\right\|\right),
\end{aligned}
$$

where $G_{i}: \mathbb{R}^{p} \rightarrow \mathbb{R}^{s}, s \geq 1$ for $i=1, \ldots, r$, are linear operators, $\|$.$\| is the \ell_{2}$ norm, $\varphi: \mathbb{R}_{+}=\{t \mid t \geq 0\} \rightarrow$ $\mathbb{R}$ is called a potential function $(\mathrm{PF})$ and $\beta>0$ is a parameter. Typically ${ }^{1}$, either $s=1$ and operators $G_{i}$

\footnotetext{
${ }^{*}$ Centre de Mathématiques et de Leurs Applications (CNRS-UMR 8536), ENS de Cachan, 61 avenue du President Wilson, 94235 Cachan Cedex, France (nikolova@cmla.ens-cachan.fr).

${ }^{\dagger}$ Department of Mathematics, The Chinese University of Hong Kong, Shatin, Hong Kong (rchan@math.cuhk.edu.hk). This work was supported by HKRGC Grant CUHK 400503 and CUHK DAG 2060257.
} 
give rise to finite differences between neighboring samples of $x$, or $s=2$ and every $G_{i}$ yields a discrete approximation of the gradient of $x$ at $i$. One can observe that $s=1$ is used along with Markov random field models for Bayesian inference, e.g. [6, 1, 7, 8, 9, 10], while $s=2$ along with variational formulations, e.g. $[11,12,13,14,3]$. The rationale of the image and signal restoration approach (1)-(2) has widely been discussed in the literature, let us evoke $[4,2,1,6,9,3]$ among many others. Let $G$ denote the $r s \times p$ matrix obtained by vertical concatenation of the matrices $G_{i}$, that is $G=\left[G_{1}^{T}, \ldots, G_{r}^{T}\right]^{T}$. A basic condition in order to have regularization is that [15]

$$
\operatorname{Ker} A^{T} A \cap \operatorname{Ker} G^{T} G=\{0\} .
$$

Many different functions $\varphi$ can be found in the literature $[6,16,9,13,17,3]$, some of the most popular ones are given in Table 1. Observe that all these PFs satisfy the general condition below:

$\mathbf{H 1} \varphi$ is continuous and increasing on $\mathbb{R}_{+}$with $\varphi \not \equiv 0$ and $\varphi(0)=0$.

The effect of the choice of $\varphi$ on the solution has been discussed e.g. in [17, 16, 13, 18, 19]. One important requirement is that $\varphi$ allows large differences ("edges") to be recovered in the solution $\hat{x}$. From the references cited above, it is well known that edge-preserving functions are nearly affine beyond a neighborhood of the origin. The computation of a minimizer $\hat{x}$ of $J$ then presents a real challenge since $\varphi^{\prime}$ involves almost flat regions, in addition to the high dimensionality of the problem and the usual ill-conditioning of $A^{T} A$.

This paper focuses precisely on the computation of a minimizer $\hat{x}$ of $J$ when $\operatorname{PF} \varphi$ is a smooth edgepreserving function. In their inaugural paper [8], Geman and Reynolds have shown that $\hat{x}$ is also given by

$$
(\hat{x}, \hat{b})=\arg \min _{(x, b)} \mathcal{J}(x, b),
$$

where $b \in \mathbb{R}_{+}^{r}$ is an auxiliary variable (called also a line process) and $\mathcal{J}$ is of the form

$$
\mathcal{J}(x, b)=\|A x-y\|^{2}+\beta \sum_{i=1}^{r}\left(\frac{b_{i}}{2}\left\|G_{i} x\right\|^{2}+\psi\left(b_{i}\right)\right)
$$

for $\psi: \mathbb{R}_{+} \rightarrow \mathbb{R}$ a function outlined in Section 2. One can notice that $x \rightarrow \mathcal{J}(x, b)$ is quadratic and that $b \rightarrow \mathcal{J}(x, b)$ can be minimized separately for each $b_{i}$. Then a two-step alternate minimization scheme is used: if the $(k-1)$ th iterate is $\left(b^{(k-1)}, x^{(k-1)}\right)$, the next one is defined by

$$
\begin{aligned}
& b^{(k)}=\arg \min _{b} \mathcal{J}\left(x^{(k-1)}, b\right), \\
& x^{(k)}=\arg \min _{x} \mathcal{J}\left(x, b^{(k)}\right) .
\end{aligned}
$$

The resulting minimization method is called half-quadratic. It can also be said multiplicative ${ }^{2}$ because the line variables $b_{i}$ in (4) multiply the relevant differences $G_{i} x$. Half-quadratic minimization has been

\footnotetext{
${ }^{1}$ Let $x$ be an $m \times n$ image. If $s=1$, for every $(i, j) \in\{1, \ldots, m\} \times\{1, \ldots, n\}$, there are associated two, or possibly four operators $G_{i}$ that yield the differences $x_{i, j}-x_{i-1, j}$ and $x_{i, j}-x_{i, j-1}$, and possibly also $x_{i, j}-x_{i-1, j+1}$ and $x_{i, j}-x_{i+1, j-1}$. When $s=2$, for every $(i, j)$ the operator $G_{i, j}$ is usually defined by $G_{i, j} x=\left[x_{i, j}-x_{i-1, j}, x_{i, j}-x_{i, j-1}\right]^{T}$. The operators relevant to the boundaries are defined according to the boundary conditions.

${ }^{2}$ Let us mention that another form of half-quadratic minimization, where $b$ is involved additively via terms of the form $\left(b_{i}+g_{i}^{T} x\right)^{2}$, was initiated in [10] and studied e.g. in [20,24, 23, 15]; it is out of the scope of this paper.
} 


\begin{tabular}{|c|c|c|c|}
\hline & Convex PFs & & Nonconvex PFs \\
\hline (f1) & $t^{\alpha}, 1<\alpha \leq 2$ & (f6) & $1-\exp \left(-\alpha t^{2}\right)$ \\
\hline (f2) & $\sqrt{\alpha+t^{2}}$ & (f7) & $\frac{\alpha t^{2}}{1+t^{2}}$ \\
\hline $\begin{array}{l}(\mathrm{f} 3) \\
(\mathrm{f} 4)\end{array}$ & $\begin{array}{l}\log (\cosh (\alpha t)) \\
t / \alpha-\log (1+t / \alpha)\end{array}$ & $\begin{array}{l}\text { (f8) } \\
\text { (f9) }\end{array}$ & $\begin{array}{l}\log \left(\alpha t^{2}+1\right) \\
\min \left\{\alpha t^{2}, 1\right\}\end{array}$ \\
\hline (f5) & $\left\{\begin{array}{lll}\frac{t^{2}}{2} & \text { if } \quad 0 \leq t \leq \alpha \\
\alpha|t|-\frac{\alpha^{2}}{2} & \text { if } \quad t>\alpha\end{array}\right.$ & (f10) & $\begin{cases}\sin \left(\alpha t^{2}\right) & \text { if } \quad 0 \leq t \leq \sqrt{\frac{\pi}{2 \alpha}} \\
1 & \text { if } t>\sqrt{\frac{\pi}{2 \alpha}}\end{cases}$ \\
\hline
\end{tabular}

Table 1: Commonly used functions $\varphi: \mathbb{R}_{+} \rightarrow \mathbb{R}$ where $\alpha>0$ is a parameter and $\varphi^{\prime}(0)=0$.

considered in a large number of papers ${ }^{3}$; e.g. [20, 13, 21, 14, 22, 23]. Connections of half-quadratic method with other well-known methods have also been explored, most notably, with generalized Weiszfeld's method [25], with statistical EM algorithms [26], with Lagrangian unconstrained optimization in recursive robust fitting [27], with quasi-Newton minimization [15, 28] and as a residual steepest descent method [28].

The contribution of this paper is to show that the half-quadratic minimization defined by (4)-(5) is equivalent to the very classical gradient linearization approach, known also as the relaxed fixed point iteration: in order to solve the equation $\nabla J(\hat{x})=0$, we write,

$$
\nabla J(x)=L(x) x-z .
$$

Then at each iteration $k$, one finds $x^{(k)}$ by solving the linear problem

$$
L\left(x^{(k-1)}\right) x^{(k)}=z .
$$

Connection between both approaches has been mentioned by Vogel in [29] for the particular case when $\varphi(t)=\sqrt{\alpha+t^{2}}$. As we show below, equivalence holds in general. In turn, convergence results on half-quadratic regularization can now be applied directly to the basically heuristic gradient linearization method in (7)-(8).

The outline of the paper is as follows. A concise review of the multiplicative form of half-quadratic minimization is given in Section 2. Then in Section 3 we formally show that half-quadratic minimization and the simple gradient linearization approach define exactly the same iterates. In Section 4, we give some implications of this equivalence. Conclusions are given in Section 5.

\section{Half-Quadratic Regularization (Multiplicative Form)}

To make the paper self-contained, below we present the derivation of the augmented objective $\mathcal{J}: \mathbb{R}^{\times} \mathbb{R}^{r}$ in (4) by synthesizing the results obtained in many previous papers $[8,20,13,21,14,22,23,15]$. A fundamental assumption for what follows is that

H2 $t \rightarrow \varphi(\sqrt{t})$ is concave. 
Put $\theta(t)=-\varphi(\sqrt{t})$, then $\theta$ is convex by 2 and continuous on $\mathbb{R}_{+}$by H1. Its convex conjugate [30,31] is $\theta^{*}(b)=\sup _{t \geq 0}\{b t-\theta(t)\}$ where $b \in \mathbb{R}$. Define $\psi(b)=\theta^{*}\left(-\frac{1}{2} b\right)$ which means that

$$
\psi(b)=\sup _{t \geq 0}\left\{-\frac{1}{2} b t-\theta(t)\right\}=\sup _{t \geq 0}\left\{-\frac{1}{2} b t^{2}+\varphi(t)\right\} .
$$

By the Fenchel-Moreau theorem $[30,31]$ the convex conjugate of $\theta^{*}$ satisfies $\left(\theta^{*}\right)^{*}=\theta$. Calculating $\left(\theta^{*}\right)^{*}$ at $t^{2}$ yields

$$
-\varphi(t)=\theta\left(t^{2}\right)=\sup _{b \leq 0}\left\{b t^{2}-\theta^{*}(b)\right\}=\sup _{b \geq 0}\left\{-\frac{1}{2} b t^{2}-\psi(b)\right\} .
$$

Since $\theta(t) \leq 0$ on $\mathbb{R}_{+}$, we have $\theta^{*}(b)=+\infty$ if $b>0$ and then the supremum of $b \rightarrow b t^{2}-\theta^{*}(b)$ in the middle expression above necessarily corresponds to $b \leq 0$. Finally,

$$
\varphi(t)=\inf _{b \geq 0}\left\{\frac{1}{2} b t^{2}+\psi(b)\right\} .
$$

The conclusion is that under H1 and H2, (9) holds if and only if (10) holds. This equivalence was first exhibited in [8] for nonconvex and bounded functions $\varphi$. It was established under different conditions on $\varphi$ in $[13,23,14,32,15]$.

For $\psi$ defined by (9), the function $\mathcal{J}$ in (4) clearly satisfies $J(x)=\inf _{b \in \mathbb{R}_{+}^{r}} \mathcal{J}(x, b)$ for every $x \in \mathbb{R}^{p}$ because of (10). In what follows, it is supposed that

H3 $\varphi$ is $\mathcal{C}^{1}$ on $[0,+\infty)$ with $\varphi^{\prime}(0)=0$.

Then the regularization term $\Phi$ in (2) is smooth. Furthermore, we adopt the classical assumption for edge preservation which states that $\varphi$ grows less fast than a quadratic function $[33,13,3]$ :

H4 $\lim _{t \rightarrow \infty} \varphi(t) / t^{2}=0$.

Next we focus on the possibility to achieve the supremum in (9) jointly with the infimum in (10). For any $\hat{b}>0$, define $f_{\hat{b}}: \mathbb{R}_{+} \rightarrow \mathbb{R}$ by $f_{\hat{b}}(t)=\frac{1}{2} \hat{b} t+\theta(t)$, then (9) yields $\psi(\hat{b})=-\inf _{t \geq 0} f_{\hat{b}}(t)$. Observe that $f_{\hat{b}}$ is convex by $\mathrm{H} 2$ with $f_{\hat{b}}(0)=0$ by $\mathrm{H} 1$ and $\lim _{t \rightarrow \infty} f_{\hat{b}}(t)=+\infty$ by H4, hence $f_{\hat{b}}$ has a unique minimum reached at a $\hat{t} \geq 0$. According to $(9), \psi(\hat{b})=-\frac{1}{2} \hat{b} \hat{t}^{2}+\varphi(\hat{t})$, then equivalently the infimum in (10) is reached for $\hat{b}$ since $\varphi(\hat{t})=\frac{1}{2} \hat{b} \hat{t}^{2}+\psi(\hat{b})$. Then one finds ${ }^{4}$ that $\hat{b}=\sigma(\hat{t})$ for

$$
\sigma(t)= \begin{cases}\frac{\varphi^{\prime}(t)}{t} & \text { if } t>0, \\ \varphi^{\prime \prime}\left(0^{+}\right) & \text {if } t=0,\end{cases}
$$

where the expression for $t=0$ corresponds to $\lim _{t \backslash 0} \sigma(t)$ and uses the fact that $\varphi^{\prime}(0)=0$. Notice that by H1, $\sigma(t) \geq 0$ for all $t \geq 0$ and that by $\mathrm{H} 2, \sigma$ is decreasing on $\mathbb{R}_{+}$. Hence $\sigma(t) \in\left[0, \varphi^{\prime \prime}\left(0^{+}\right)\right]$. Let us now consider the possibility that $\hat{b}=0$. Put $\varphi_{\infty}=\lim _{t \rightarrow \infty} \varphi(t)$, then $\varphi_{\infty}=\sup _{t \geq 0} \varphi(t) \in(0, \infty]$ by H1.

\footnotetext{
${ }^{4}$ Notice that $f_{\hat{b}}^{\prime}(t)=\frac{1}{2} \hat{b}+\theta^{\prime}(t)$ is increasing on $\mathbb{R}_{+}$and that $\theta^{\prime}(t)=-\frac{\varphi^{\prime}(\sqrt{t})}{2 \sqrt{t}}$. If $f_{\hat{b}}^{\prime}\left(0^{+}\right) \geq 0$, i.e. if $\hat{b} \geq \varphi^{\prime \prime}\left(0^{+}\right), f_{\hat{b}}$ reaches its minimum at $\hat{t}=0$. Otherwise, its minimum is reached for a $\hat{t}>0$ such that $f_{\hat{b}}^{\prime}(\hat{t})=0$, i.e. $\hat{b}=-2 \theta^{\prime}(\hat{t})$. In this case, $t \rightarrow-\frac{1}{2} \hat{b} t^{2}+\varphi(t)$ in the last expression of (9) reaches its supremum for a $\hat{t}$ that satisfies $\hat{b}=-2 \theta^{\prime}\left(\hat{t}^{2}\right)$. Hence $(11)$.
} 
If $\varphi(t)<\varphi_{\infty}$ for all $t \geq 0$ (e.g. $\varphi$ is strictly increasing as (f1)-(f8) in Table 1 ), for $\hat{b}=0$ the term between the braces in (10) equals $\varphi_{\infty}$, so (10) cannot be realized for any finite $\hat{t} \geq 0$; then the infimum in (10) necessarily corresponds to $\hat{b}>0$. Otherwise, by H1, there is $T>0$ such that $\varphi(t)=\varphi_{\infty} \in(0, \infty)$ for all $t \geq T$, and then $\hat{b}=0$ in (9) corresponds to $\hat{t} \geq T$ in (10) and $\sigma(\hat{t})=0$ in (11) since $\varphi^{\prime}(t)=0$ if $t \geq T$ (e.g. (f9) in Table 1).

The formula in (11) was initially obtained in [13] under special conditions on $\varphi$ and was considered in later papers, e.g. $[14,23,15]$. The derivation presented above is more general than these references. Let us resume its meaning: if $H 1-H_{4}$ hold, for every $t \geq 0$, the infimum in (10) is reached for $\hat{b}=\sigma(t) \in$ $\left[0, \varphi^{\prime \prime}\left(0^{+}\right)\right]$, where $\sigma$ in defined in (11), and we have $\hat{b}>0$ unless there is a $T>0$ such that $\varphi(t)=$ const if $t \geq T$.

Let us come back to the augmented objective function $\mathcal{J}$ given in (4). The result given in (11) shows that $J(x)=\mathcal{J}(x, \hat{b})$ if $\hat{b}_{i}=\sigma\left(\left\|G_{i} \hat{x}\right\|\right)$ for all $i \in\{1, \ldots, r\}$. For convenience, let us now write $\mathcal{J}$ in (4) as

$$
\mathcal{J}(x, b)=\|A x-y\|^{2}+\frac{\beta}{2}(G x)^{T} \mathcal{D}(b) G x+\beta \sum_{i=1}^{r} \psi\left(b_{i}\right),
$$

where for every $b \in \mathbb{R}_{+}^{r}$,

$$
\mathcal{D}(b)=\operatorname{diag}\left(b_{1} \mathbb{1}_{s}, \ldots, b_{r} \mathbb{1}_{s}\right) \text { for } \mathbb{1}_{s}=[1, \ldots, 1]^{T} \in \mathbb{R}^{s} .
$$

Define $\mathcal{L}: \mathbb{R}_{+}^{r} \rightarrow \mathbb{R}^{p \times p}$ by

$$
\mathcal{L}(b)=2 A^{T} A+\beta G^{T} \mathcal{D}(b) G .
$$

Sufficient conditions for the invertibility of $\mathcal{L}(b)$ are that $b \in(0, \infty)^{r}$ and $(3)$ holds, or that $\operatorname{rank} A=p$. Henceforth we assume that $\mathcal{L}$ is invertible on the domain of $b$, namely $\left\{\sigma(t): t \in \mathbb{R}_{+}\right\}^{r}$. Combining (11) with the necessary and sufficient condition for a minimum of $x \rightarrow \mathcal{J}(x, b)$ shows that the minimum of $\mathcal{J}$ is characterized by

$$
\begin{aligned}
& \hat{b}_{i}=\sigma\left(\left\|G_{i} \hat{x}\right\|\right), \quad 1 \leq i \leq r, \\
& \mathcal{L}(\hat{b}) \hat{x}=2 A^{T} y .
\end{aligned}
$$

The alternate minimization scheme mentioned in (5)-(6) is aimed at solving (14)-(15). Given $x^{(k-1)}$, the first step of iteration $k$, as defined in (5), has an explicit form:

$$
b_{i}^{(k)}=\sigma\left(\left\|G_{i} x^{(k-1)}\right\|\right), \quad i=1, \ldots, r .
$$

Its second step, defined by (6), amounts to

$$
x^{(k)}=\left(\mathcal{L}\left(b^{(k)}\right)\right)^{-1} 2 A^{T} y .
$$

The convergence of the resulting iterative scheme (16)-(17) was considered under different assumptions on $\varphi$ in $[13,21,23]$ while its speed was analyzed in [19, 28].

It is worth to recall the interpretation of the auxiliary array $b$ pioneered in [8]. Since $\sigma$ in (11) is decreasing on $\mathbb{R}_{+},(14)$ shows that a large $\hat{b}_{i}$ is attached to a small $\left\|G_{i} \hat{x}\right\|$ and vice versa. In this way, 
each $\hat{b}_{i}$ expresses the weight of the quadratic smoothness constraint imposed to the relevant $G_{i} \hat{x}$. Indeed, the auxiliary variable $b$ has been interpreted by Geman and Reynolds [8] as a continuous-valued, noninteracting line process. This interpretation had a significant impact on the research on edge-preserving regularization during the last 15 years. From the PDE point of view, the auxiliary variables $b_{i}, 1 \leq i \leq r$, can also be seen as the conduction coefficients in an anisotropic diffusion equation, see [12,34] for details.

\section{Linearization of the Gradient}

A natural alternative to simplify the search for a solution to $\nabla J(x)=0$ is to linearize this gradient at each step, as sketched in (7)-(8). Below we develop these expressions for an objective function $J$ of the form (1)-(2). The gradient of $J$ reads

$$
\nabla J(x)=2 A^{T} A x+\beta \sum_{i} G_{i}^{T} \frac{\varphi^{\prime}\left(\left\|G_{i} x\right\|\right)}{\left\|G_{i} x\right\|} G_{i} x-2 A^{T} y .
$$

Using that $x \rightarrow G_{i} x /\left\|G_{i} x\right\|$ is uniformly bounded on $\mathbb{R}^{p}$ and that $\varphi^{\prime}(0)=0$, we can write that

$$
\frac{\varphi^{\prime}\left(\left\|G_{i} x\right\|\right)}{\left\|G_{i} x\right\|} G_{i} x=\left\{\begin{array}{lll}
0 & \text { if } & G_{i} x=0 \\
\sigma\left(\left\|G_{i} x\right\|\right) G_{i} x & \text { else. }
\end{array}\right.
$$

where $\sigma$ is the function in (11). Notice that the two cases in (19) are necessary because if $\sigma(0)=+\infty$, which occurs for instance for $\varphi(t)=t^{\alpha}, 1<\alpha<2$, we can replace this infinite value by any positive real number without changing the expression in (18). Using (19), $\nabla J$ in (18) reads

$$
\begin{aligned}
\nabla J(x) & =2 A^{T} A x+\beta G^{T} \mathcal{D}\left(\left[\sigma\left(\left\|G_{i} x\right\|\right)\right]_{i=1}^{r}\right) G x-2 A^{T} y \\
& =\left(2 A^{T} A+\beta G^{T} \mathcal{D}\left(\left[\sigma\left(\left\|G_{i} x\right\|\right)\right]_{i=1}^{r}\right) G\right) x-2 A^{T} y
\end{aligned}
$$

where $b \rightarrow \mathcal{D}(b)$ is the application defined by (12). Then $\nabla J(x)$ is easily put into the form (7), namely $\nabla J(x)=L(x) x-z$, for

$$
\begin{aligned}
L(x) & =\mathcal{L}\left(\left[\sigma\left(\left\|G_{i} x\right\|\right)\right]_{i=1}^{r}\right), \\
z & =2 A^{T} y,
\end{aligned}
$$

where $\mathcal{L}$ is the matrix-valued function defined in (13). The matrix $L(x)$ is invertible if $\mathcal{L}(b)$ is invertible on the domain of $b$, a question which was addressed already in Section 2. Applying (8) actually yields

$$
x^{(k)}=\left(\mathcal{L}\left(\left[\sigma\left(\left\|G_{i} x^{(k-1)}\right\|\right)\right]_{i=1}^{r}\right)\right)^{-1} 2 A^{T} y .
$$

This amounts to inserting the expression for the $b_{i}^{(k)}$ in (16) into the expression for $x^{(k)}$ in (17) in the half-quadratic minimization methods. It follows that the half-quadratic minimization and the gradient linearization approach construct exactly the same sequence of iterates $x^{(k)}$.

The gradient linearization method was used by Vogel and Oman in [35, 29] to minimize an approximate total variation regularization corresponding to $\varphi(t)=\sqrt{\alpha+t^{2}} \approx|t|$ and it was called the "lagged diffusivity fixed point iteration". In [29] the authors mention that it amounts to apply the multiplicative form of half-quadratic minimization to this $\varphi$. As we have shown above, the equivalence holds for general functions $\varphi$ applied either to the $\ell_{1}$ or to the $\ell_{2}$ norm of $G_{i} x$. 


\section{Implications}

The equivalence established in the last section shows in particular that the gradient linearization iteration is convergent for all objective functions for which convergence of half-quadratic regularization has been proven $[13,21,23]$. We can also use the equivalence to connect the half-quadratic minimization to other well-known methods. As an example, it has been pointed out in $[15,28]$ that for convex PFs $\varphi$, the halfquadratic minimization corresponds to a quasi-Newton minimization. The same holds now in our more general context since any gradient linearization method can always be viewed as a form of quasi-Newton method. Indeed, starting with (21), we derive

$$
\begin{aligned}
x^{(k)} & =\left(L\left(x^{(k-1)}\right)\right)^{-1} z \\
& =\left(L\left(x^{(k-1)}\right)\right)^{-1}\left(L\left(x^{(k-1)}\right) x^{(k-1)}-L\left(x^{(k-1)}\right) x^{(k-1)}+z\right) \\
& =x^{(k-1)}-\left(L\left(x^{(k-1)}\right)\right)^{-1} \nabla J\left(x^{(k-1)}\right),
\end{aligned}
$$

where in the last equality, we use (7). This shows that half-quadratic regularization (16)-(17), or equivalently the gradient linearization iteration (21), performs a quasi-Newton iteration where the Hessian of $J$ at $x^{(k-1)}$ is approximated by $L\left(x^{(k-1)}\right)$ as defined in (20).

Following [25, 28], we note further that if one defines

$$
G(y, x)=J(x)+(y-x)^{T} \nabla J(x)+\frac{1}{2}(y-x)^{T} L(x)(y-x),
$$

then $G(y, x)$ provides a majorizing quadratic approximation for $F(x)$. In the generalized Weiszfeld's algorithm, the iterates are given by $x^{(k)}=\arg \min _{x} G\left(x, x^{(k-1)}\right)$ which amounts to the gradient linearization method (21) and equivalently to the half-quadratic minimization (16)-(17) whenever $L$ is given by (20).

\section{Conclusions}

Half-quadratic minimization has been studied and used by numerous authors. In this paper we have shown that it is equivalent to the very basic approach where at each iteration a linear approximation of the gradient is used. We also demonstrate that it is a form of quasi-Newton method and is closely related to the gradient descent method and the generalized Weiszfeld's algorithm. This paper nicely shows a case where research follows a tortuous way to find a simple result.

\section{References}

[1] G. Demoment, "Image reconstruction and restoration : Overview of common estimation structure and problems", IEEE Transactions on Acoustics Speech and Signal Processing, vol. ASSP-37, no. 12, pp. 2024-2036, Dec. 1989.

[2] A. Tarantola, Inverse problem theory : Methods for data fitting and model parameter estimation, Elsevier Science Publishers, Amsterdam, 1987. 
[3] G. Aubert and P. Kornprobst, Mathematical problems in images processing, Springer-Verlag, Berlin, 2002.

[4] A. Tikhonov and V. Arsenin, Solutions of Ill-Posed Problems, Winston, Washington DC, 1977.

[5] S. Geman and D. Geman, "Stochastic relaxation, Gibbs distributions, and the Bayesian restoration of images", IEEE Transactions on Pattern Analysis and Machine Intelligence, vol. PAMI-6, no. 6, pp. 721-741, Nov. 1984.

[6] J. E. Besag, "Digital image processing : Towards Bayesian image analysis", Journal of Applied Statistics, vol. 16, no. 3, pp. 395-407, 1989.

[7] D. Geman, Random fields and inverse problems in imaging, vol. 1427, pp. 117-193, École d'Été de Probabilités de Saint-Flour XVIII - 1988, Springer-Verlag, lecture notes in mathematics edition, 1990.

[8] D. Geman and G. Reynolds, "Constrained restoration and recovery of discontinuities", IEEE Transactions on Pattern Analysis and Machine Intelligence, vol. PAMI-14, no. 3, pp. 367-383, Mar. 1992.

[9] C. Bouman and K. Sauer, "A generalized Gaussian image model for edge-preserving MAP estimation", IEEE Transactions on Image Processing, vol. 2, no. 3, pp. 296-310, July 1993.

[10] D. Geman and C. Yang, "Nonlinear image recovery with half-quadratic regularization", IEEE Transactions on Image Processing, vol. IP-4, no. 7, pp. 932-946, July 1995.

[11] A. Blake and A. Zisserman, Visual reconstruction, The MIT Press, Cambridge, 1987.

[12] P. Perona and J. Malik, "Scale-space and edge detection using anisotropic diffusion", IEEE Transactions on Pattern Analysis and Machine Intelligence, vol. PAMI-12, pp. 629-639, July 1990.

[13] P. Charbonnier, L. Blanc-Féraud, G. Aubert, and M. Barlaud, "Deterministic edge-preserving regularization in computed imaging", IEEE Transactions on Image Processing, vol. 6, no. 2, pp. 298-311, Feb. 1997.

[14] S. Teboul, L. Blanc-Féraud, G. Aubert, and M. Barlaud, "Variational approach for edge-preserving regularization using coupled pde's", IEEE Transactions on Image Processing, vol. 7, no. 3, pp. 387-397, Mar. 1998.

[15] M. Nikolova and M. Ng, "Analysis of half-quadratic minimization methods for signal and image recovery", SIAM Journal on Scientific Computing, vol. 27, no. 3, pp. 937-966, 2005.

[16] M. Black and A. Rangarajan, "On the unification of line processes, outlier rejection, and robust statistics with applications to early vision", International Journal of Computer Vision, vol. 19, no. 1, pp. 57-91, 1996. 
[17] S. Li, Markov Random Field Modeling in Computer Vision, Springer-Verlag, New York, 1 edition, 1995.

[18] M. Nikolova, "Weakly constrained minimization. Application to the estimation of images and signals involving constant regions", Journal of Mathematical Imaging and Vision, vol. 21, no. 2, pp. 155-175, Sep. 2004.

[19] M. Nikolova, "Analysis of the recovery of edges in images and signals by minimizing nonconvex regularized least-squares", SIAM Journal on Multiscale Modeling and Simulation, vol. 4, no. 3, pp. 960-991, 2005.

[20] P. Charbonnier, L. Blanc-Féraud, G. Aubert, and M. Barlaud, "Two deterministic half-quadratic regularization algorithms for computed imaging", in Proceedings of IEEE ICIP, 1994, vol. 2, pp. $168-172$.

[21] A. H. Delaney and Y. Bresler, "Globally convergent edge-preserving regularized reconstruction: an application to limited-angle tomography", IEEE Transactions on Image Processing, vol. 7, pp. 204-221, Feb. 1998.

[22] P. Kornprobst, R. Deriche, and G. Aubert, "Image sequence analysis via partial differential equations", Journal of Mathematical Imaging and Vision, vol. 11, no. 1, pp. 5-26, Oct. 1999.

[23] J. Idier, "Convex half-quadratic criteria and auxiliary interacting variables for image restoration", IEEE Transactions on Image Processing, vol. 10, no. 7, pp. 1001-1009, July 2001.

[24] G. Aubert and L. Vese, "A variational method in image recovery", SIAM Journal on Numerical Analysis, vol. 34, no. 5, pp. 1948-1979, 1997.

[25] T. Chan and P. Mulet, "On the convergence of the lagged diffusivity fixed point method in total variation image restoration", SIAM Journal on Numerical Analysis, vol. 36, no. 2, pp. 354-367, 1999.

[26] F. Champagnat and J. Idier, "A connection between half-quadratic criteria and em algorithms", IEEE Signal Processing Letters, vol. 11, no. 9, pp. 709-712, Sep. 2004.

[27] J.-P. Tarel, S.-S. Ieng, and P. Charbonnier, "Using robust estimation algorithms for tracking explicit curves", in ECCV. LNCS 2350, Springer-Verlag, 2002, pp. 492-507.

[28] M. Allain, J. Idier, and Y. Goussard, "On global and local convergence of half-quadratic algorithms", IEEE Transactions on Image Processing, vol. 15, no. 5, pp. 1130-1142, 2006.

[29] C. R. Vogel and M. E. Oman, "Fast, robust total variation-based reconstruction of noisy, blurred images", IEEE Transactions on Image Processing, vol. 7, no. 6, pp. 813-824, Mar. 1998.

[30] R. T. Rockafellar, Convex Analysis, Princeton University Press, 1970. 
[31] H. Brezis, Analyse fonctionnelle, Collection mathématiques appliquées pour la maîtrise. Masson, Paris, 1992.

[32] P. Ciuciu and J. Idier, "A half-quadratic block-coordinate descent method for spectral estimation", Signal Processing, , no. 82, pp. 941-959, 2002.

[33] S. Z. Li, "On discontinuity-adaptive smoothness priors in computer vision", IEEE Transactions on Pattern Analysis and Machine Intelligence, vol. PAMI-17, no. 6, pp. 576-586, June 1995.

[34] L. Blanc-Féraud, P. Charbonnier, G. Aubert, and M. Barlaud, "Nonlinear image processing: modelling and fast algorithm for regularisation with edge detection", in Proceedings of IEEE ICIP, 1995, vol. 2, pp. $474-477$.

[35] C. R. Vogel and M. E. Oman, "Iterative method for total variation denoising", SIAM Journal on Scientific Computing, vol. 17, no. 1, pp. 227-238, 1996. 